\title{
Route Planning of Unmanned Aerial Vehicles under Recharging and Mission Time Constraints
}

\author{
Kriangsak Phalapanyakoon \\ Department of Computer Engineering, \\ King Mongkut's University of Technology, Thonburi, Bangkok, Thailand. \\ Corresponding author: kriangsak.indy117@mail.kmutt.ac.th \\ Peerapon Siripongwutikorn \\ Department of Computer Engineering, \\ King Mongkut's University of Technology, Thonburi, Bangkok, Thailand. \\ E-mail: peerapon.sir@mail.kmutt.ac.th
}

(Received on May 6, 2021; Accepted on September 27, 2021)

\begin{abstract}
This paper investigates the problem of route planning for rechargeable unmanned aerial vehicles (UAV) under the mission time constraint in cases where more than one trip per round is required due to limited battery capacities. The goal is to determine the number of UAVs to be deployed and the flying paths that minimize the total mission cost. Unlike previous works, the electric cost incurred by UAV recharging proportional to actual flying distances is incorporated into our model. The problem is formulated as a mixed-integer programming model to minimize the sum of electric charging cost, the UAV usage cost, and the penalty cost from the violation of the mission time constraint. Extensive numerical experiments are conducted to examine the integrity and performance of the proposed model under various model parameters and deployment scenarios in grid areas and a real terrain area. The optimal solutions can be obtained for small-scale problem instances in a reasonable runtime. For large-scale problems, only feasible solutions can be obtained due to limited computational resources.
\end{abstract}

Keywords- Unmanned aerial vehicle (UAV), Route planning, Rechargeable unmanned aerial vehicles, Mission time constraint, Mixed-integer programming (MIP).

\section{Introduction}

Due to the rapid development of transportation technology over the past few years, unmanned aerial vehicles (UAVs) have proven beneficial in terms of cost and time efficiency, especially in areas inaccessible to humans (Joshi, 2019). From deliveries at the rush hour, express shipping, delivering life-sustaining bags in flood areas, storm tracking, hurricane forecasting, or unmanned cargo transport, UAV use improves efficiency and raises productivity while reducing the investment cost, workload, and operating time. Due to limited fleet size and limited battery capacity, UAVs may have to operate multiple trips and require recharging in between to complete the mission in a large service area. Non-optimal planning of the flying paths can result in an unnecessarily high cost from UAV charging and usages and excessive time in completing missions. Thus, the model of route planning for rechargeable UAVs is crucial to obtain the optimal solution with minimum total mission cost.

In practice, UAV recharging incurs an electricity cost that depends on the remaining UAV energy before recharging when returning to the base, and the recharging time adds to the total mission time. For the same coverage area, deploying more UAVs will shorten the mission time and results in less charging time at the expense of a higher UAV usage cost. A time constraint is specified for 
time-critical missions, and the amount of completion time exceeding the time constraint is penalized. Trade-offs among UAV recharging during trips, the number of UAVs deployed, and the mission time regarding the total mission cost in the UAV route planning problem have not been considered in prior works. Our objective is to determine the UAV flying paths that minimize the total mission cost from these three cost components.

The major contributions of this research are two fold:

- First, we formulate and solve the mathematical model for the route planning of multiple rechargeable UAVs with multiple trips and rounds under the mission time constraint that minimizes the total operating cost. Unlike models in previous works, we incorporate the recharging period based on the actual remaining UAV energy. The proposed model determines the number of UAVs to be deployed and their flying paths that minimize the sum of three cost components: recharging cost, UAV usage, and penalty of mission time violation.

- Second, the effects of the problem size and model parameters are explored for the model's computational limitations. A sensitivity analysis of parameters was also conducted. A real terrain is also used as the problem input to demonstrate practical usage of the model.

The remainder of this paper is organized as follows. Section 2 reviews related works on UAV route planning. In Section 3, the problem description, assumptions, notations, and mathematical models are presented. Section 4 discusses the computational results from our model and the sensitivity to the model parameters. Finally, in Section 5, we summarize our conclusion and provide directions for future research.

\section{Related Works}

In cargo transportation research, a basic yet essential problem is to seek an optimal path for a fleet of vehicles, known as the vehicle routing problem (VRP). Given a set of known locations, a fleet of vehicles starts from a depot and visits all locations, then returns to the depot or the base under a set of truck capacity and delivery time window constraints (Dantzig and Ramser, 1959). The time window constraint is crucial in the mission of last-mile delivery. In the VRP with time windows (VRPTW), the time window constraint can be violated if the penalty is paid and hence is considered as a soft time window. Troudi et al. (2018) presented an analytical model of a UAV delivery mission that considers the autonomy and energy consumption based on the UAV specification. Their objectives were to minimize the total distance, the total number of UAVs used, and the total battery used during the mission. Kitjacharoenchai and Lee (2019) and Han et al. (2020) considered the last-mile delivery that uses a truck to store goods and uses UAVs to deliver goods to customers. Kitjacharoenchai and Lee's work focused on minimizing the total arrival time of a truck and UAVs after completing the mission, while Han's work implemented the artificial bee colony to minimize the total energy consumption of a truck and UAVs. The minimization of the total number of UAVs was also considered in their work. The works mentioned above considered only a single trip, and hence their models do not take the battery or fuel capacity constraints into account.

The multiple-trip vehicle routing problem (MTVRP) is an extension of classical VRP, where each vehicle is allowed to perform multiple trips if it cannot visit all points of interest within one trip 
(Zmazek et al., 2005). Heuristics such as tabu search by Alonso et al. (2008) and genetic algorithm by Ayadi and Benadada (2013) have been implemented to solve the basic MTVRP. Kabcome and Mouktonglang (2015) proposed the mathematical model to solve VRP with a soft time window, multiple product categories, including multiple compartments and trips. Zhen et al. (2020) defined the multi-depot multi-trip VRP with time window and release date for the lastmile distribution scenarios. They implemented the CPLEX solver for small-scale instances and developed a hybrid particle swarm optimization algorithm and hybrid genetic algorithm to solve large-scale instances. However, these works did not consider the energy constraint of vehicles and the cost of deploying vehicles.

For the recharging vehicle routing problem (RVRP), (electric) vehicles with limited range or energy travel over a predefined set of points of interest and can recharge at destinations to continue a tour, or vehicles can be recharged at the depot only (Conrad and Figliozzi, 2011). Penna et al. (2016) proposed a hybrid iterative local search algorithm for the VRP with time window and recharging stations, where the objective is to determine the composition of the fleet that minimizes the fixed and travel cost. Hiermann et al. (2016) implemented a hybrid heuristic that combines adaptive large neighborhood search with an embedded local search and labeling procedure to solve the problem. To recharge energy-limited UAVs to visit a set of sites, Yu et al. (2019) presented the algorithm that finds the order of site visits and where to land to recharge either on a stationary recharging station or unmanned ground vehicles (UGVs) in the least amount of time. A time-dependent fleet of heterogeneous UAVs routing problem which considers multiple charging stations and operational requirements was considered in Coelho et al. (2017). However, these works only considered a single trip and did not include the cost of deploying UAVs in their models. So, to visit all locations, the combination of multiple trips with battery recharging must be integrated into our proposed model.

Unlike terrestrial vehicles such as trucks, trains, or public transportation, UAVs have a small battery capacity, limiting their flight duration and distance. For a large deployment area, one must strike a balance between the cost of deploying many UAVs and the cost of recharging. Dorling et al. (2017) presented multi-trip VRP for UAV delivery that considered the effect of payload weight on the energy consumption subject to delivery time or budget constraints. However, battery swapping on UAVs was used instead of recharging and the UAV usage cost was not considered. The work by Choi et al. (2019) studied a multi-trip VRP for small UAV-based urban delivery. The model minimized the sum of acquisition cost and the operating cost of the mission and considered the package reloading time at the depot.

From the works discussed earlier, the research gap is the lack of UAV route planning that considers the battery consumption and recharging during the trips, as well as the cost of deploying UAVs in the model. The battery recharging time based on the remaining energy or the flying distance of UAVs has not previously been considered, and no research exists that integrates the battery recharging time, the UAV usage cost, and the penalty of mission time violation in UAV route planning. Our objective is to determine the optimal UAV flying paths and number of UAVs to be deployed under multiple trips and rounds that minimizes the total mission cost. Unlike the previous works that have been discussed, we consider the cost of UAV recharging that depends on the flying distance, the UAV usage cost and the penalty of mission time violation in UAV route planning. 


\section{Proposed Model}

This section formally defines the problem of route planning for UAVs with multiple rounds and trips, mission time constraint, and battery recharging. Then, the mixed-integer programming (MIP) model for the problem is developed.

\subsection{Problem Description and Assumptions}

Consider a set of locations or coordinates to be visited by UAVs, as in survival bag delivery under flood situations in an unreachable area for the rescue team. Many delivery rounds may be required for the whole mission, where all the locations have been visited once in a single round. The whole mission deploys the number of UAVs that will be released from the same base location, and all the UAVs return to the base location when they finish. Since each UAV has a limited battery capacity, it may repeatedly fly back to the base location for battery recharging as needed and resume its operations to finish all its assigned locations. The decision for recharging is made based on the percentage of UAV remaining battery capacity as we certainly cannot let a UAV run out of battery airborne. We call it a trip when a UAV departs and returns to the base location, and the sequence of locations to be visited by a UAV for the whole mission as a route. So, the route of a UAV can consist of multiple trips with recharging periods in between as shown in Figure 1.

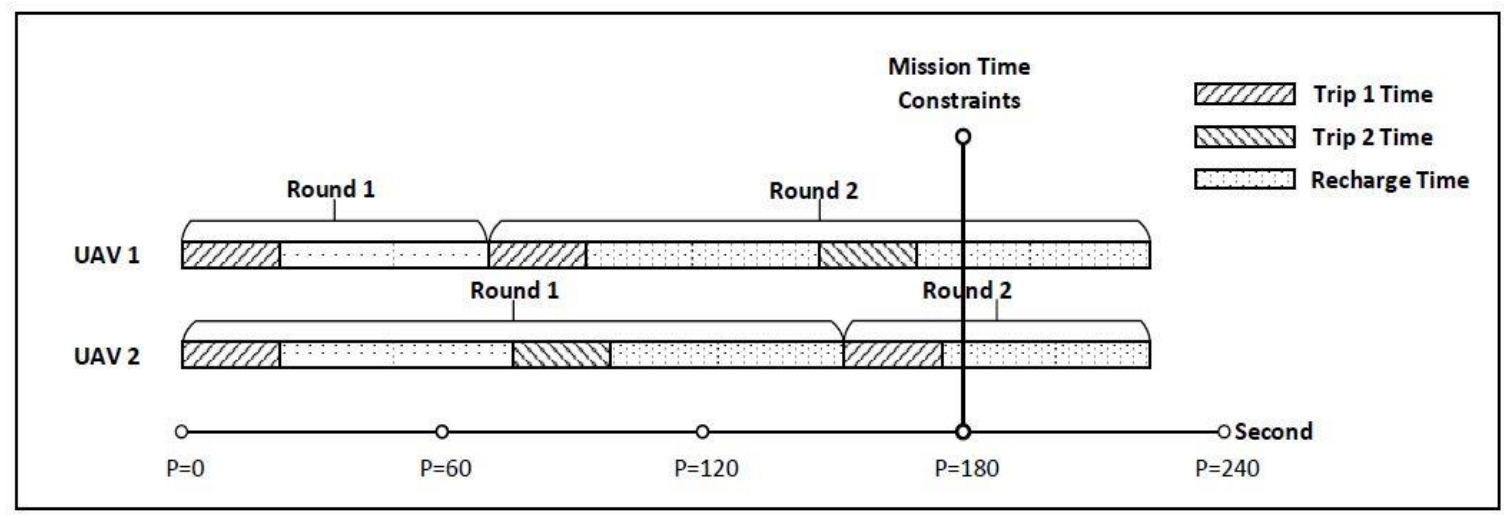

Figure 1. An example of timing diagrams of two rechargeable UAVs.

The total cost incurred by the mission is the sum of UAV electric or recharging usage cost, UAV usage cost, and time penalty cost when the time taken to complete the whole mission exceeds a pre-specified time constraint. Our goal is to determine routes of the UAVs to minimize the total cost under the mission time constraints. The time penalty cost unit can be applied to weigh the impact of a time constraint violation, which is application specific. The following assumptions are made in our problem:

- All the UAVs are homogeneous so that they have the same energy consumption rate, flying speed, and battery capacity.

- The UAV energy consumption linearly increases with the flying distance.

- Each UAV trip starts and ends at the base location.

- Each location, except the base location, must be visited at least once in each round. 
- At the end of each round, each UAV recharges its battery before performing another round.

- The batteries of all UAVs must be at the full level at the beginning and end of the mission.

\subsection{Model Formulation}

\subsubsection{Notations}

We formulate the UAV route planning problem as an MIP model. The set of locations is defined as a directed graph $G=(\mathbb{V}, \mathbb{E})$, where $\mathbb{V}$ is a set of vertices or locations $\{1,2, \ldots, N\}$ with known coordinates and location 0 denotes the base location, and $\mathbb{E}=\{(i, j) \mid i, j \in \mathbb{V}\}$ is a set of edges among neighboring vertices. The neighboring locations of $i \in \mathbb{V}$ are the smallest set of locations forming a convex hull with $i$ inside. The Euclidean distance between two locations $i$ and $j$ is denoted by $d_{i j}$. Denote the number of UAVs as $N_{U}=|\mathbb{U}|$. Each UAV $u$ has a battery capacity of $Q$. A trip $t$ is defined by a sequence of visits to locations and a set of trips by $\mathbb{T}$ with a maximum allowed trip of $T_{\max }$ as $\mathbb{T}=\left\{1,2, \ldots, T_{\max }\right\}$. The set of rounds is represented by $\mathbb{R}=$ $\left\{1,2, \ldots, N_{R}\right\}$. We assume that all the UAVs fly at the constant velocity $V$ and the flying time from location $i$ to $j$ is $t_{i j}=d_{i j} / V$. A penalty cost is incurred when there exists a UAV that finishes its route after the mission time deadline or target mission time $T_{c}$, which can be chosen based on the area size, the mission purpose, and UAV capabilities. Tables 1-3 show the parameters and variables used in the formulation.

Table 1. Sets and parameters.

\begin{tabular}{|c|l|}
\hline Sets/Parameters & \\
\hline $\mathbb{V}$ & Set of locations $\{0,1,2, \ldots, N\}$ \\
\hline $\mathbb{E}$ & Set of edges $\{(i, j \mid i, j \in \mathbb{V})\}: i \neq j$ \\
\hline $\mathbb{U}$ & Set of UAVs $\left\{1,2, \ldots, N_{U}\right\}$ \\
\hline $\mathbb{T}$ & Set of trips $\left\{1,2, \ldots, T_{\max }\right\}$ \\
\hline$T_{\max }$ & Number of maximum allowed trips for each UAV \\
\hline $\mathbb{R}$ & Set of rounds $\left\{1,2, \ldots, N_{R}\right\}$ \\
\hline$Q$ & The battery capacity of each UAV (watt-hours) \\
\hline$V$ & UAV velocity (meter/second) \\
\hline$T_{c}$ & Target mission time (second) \\
\hline$M$ & Sufficiently large enough number \\
\hline$\theta$ & The limit percentage of battery capacity of each UAV in each trip \\
\hline$\alpha$ & Time penalty unit cost (baht/second) \\
\hline$\beta$ & UAV unit cost (baht/UAV) \\
\hline$\gamma$ & Energy unit cost (baht/mWh) \\
\hline$d_{i j}$ & Distance between location $i$ and $j$ (meter) \\
\hline$E_{i j}$ & Energy consumed between location $i$ and $j$ (watt-hours) \\
\hline$C R$ & The energy consumption rate of each UAV per meter (watt-hour/meter) \\
\hline$R R$ & Recharging rate of each UAV per second (seconds/watt-hours) \\
\hline
\end{tabular}


Table 2. Computation variables.

\begin{tabular}{|c|c|}
\hline Computation Variables & Description \\
\hline RemainEnergy $y^{r t u}$ & The remaining energy of each UAV at the base location in trip $t$ of round $r$ \\
\hline TotalTime $^{r t u}$ & The time consumption of each UAV in trip $t$ of round $r$ including recharging time \\
\hline RemainofUAV ${ }^{\text {ru }}$ & The remaining battery capacity at last trip in each round of UAV $u$ \\
\hline MaxTime $^{u}$ & The penalty of overtime of each UAV \\
\hline
\end{tabular}

Table 3. Decision variables.

\begin{tabular}{|l|l|}
\hline Decision Variables & Description \\
\hline$x_{i j}^{r t u}$ & Binary equals one if UAV $u$ travels from $i$ to $j$ in trip $t$ of round $r$ \\
\hline Recharge $^{r t u}$ & Binary equals one if UAV $u$ consumes energy more than $(Q \cdot \theta)$ in trip $t$ of round $r$ \\
\hline$U A V U s e d^{u}$ & Binary equals one if UAV $u$ is deployed in the mission \\
\hline$\mu_{i}^{r t u}$ & The order of location already visited by UAV $u$ before entering $i$ in trip $t$ of round $r$ \\
\hline
\end{tabular}

\subsubsection{Mathematical Model}

The problem is a minimization problem with the objective function (1) consisting of three parts. The first part is the (electric) recharging cost, which is proportional to the sum of all UAV travel distances. The second part is the cost of using UAVs. The last part is the penalty for the excess time that UAVs use beyond the mission time constraint. For the objective function to have a meaningful interpretation, appropriate values of the scaling factors $\gamma, \beta$, and $\alpha$ in the recharging cost, the UAV usage cost, and time penalty cost are used respectively so that the objective function has the monetary cost unit. So, we treat $\gamma$ as the energy unit cost we pay for every unit of electricity used during UAV recharging, $\beta$ as the UAV unit cost we pay for each UAV deployed in the mission, and $\alpha$ as the time penalty unit cost we pay for each second the target mission time has been exceeded. The value of $\alpha$ is set to reflect the degree of the time sensitivity of the mission, e.g., the one used in the mission of distributing fertilizer on a large farm should be lower than those in distributing survival bags for flood victims or extinguishing forest fire.

The proposed mathematical model consists of several constraints to handle each aspect of the problem. The constraints can be divided into three groups. The first group of constraints (2-7) is derived from standard capacitated vehicle routing problems to handle UAV routing paths. The second group of constraints (8-15) is devised to track a UAV's energy consumption and determine the amount of recharging. The third group of constraints (16-20) is to track the time spent by UAVs and determine the penalty from an excess mission time.

$\operatorname{Minimize}\left(\sum_{r \in \mathbb{R}} \sum_{t \in \mathbb{T}} \sum_{u \in \mathbb{U}} \sum_{i \in \mathbb{V}} \sum_{j \in \mathbb{V}}\left(\gamma \cdot\left(E_{i j} \cdot x_{i j}^{r t u}\right)\right)+\left(\beta \cdot \sum_{u \in \mathbb{U}} U A V U\right.\right.$ sed $\left.\left.^{u}\right)+\left(\alpha \cdot \sum_{u \in \mathbb{U}} \operatorname{MaxTime}^{u}\right)\right)$

subject to

$x_{i i}^{r t u}=0, \quad \forall i \in \mathbb{V}, \forall r \in \mathbb{R}, \forall t \in \mathbb{T}, \forall u \in \mathbb{U}$

$\sum_{t \in \mathbb{T}} \sum_{u \in \mathbb{U}} \sum_{j \in \mathbb{V}} x_{i j}^{r t u}=1, \quad \forall i \in \mathbb{V}, \forall r \in \mathbb{R}$ 
$\sum_{t \in \mathbb{T}} \sum_{u \in \mathbb{U}} \sum_{i \in \mathbb{V}} x_{i j}^{r t u}=1, \quad \forall j \in \mathbb{V}, \forall r \in \mathbb{R}$

$\sum_{j \in \mathbb{V}} x_{0 j}^{r t u}-\sum_{i \in \mathbb{V}} x_{i 0}^{r t u}=0, \quad \forall r \in \mathbb{R}, \forall t \in \mathbb{T}, \forall u \in \mathbb{U}$

$\sum_{i \in \mathbb{V}} x_{i h}^{r t u}-\sum_{j \in \mathbb{V}} x_{h j}^{r t u}=0, \quad \forall h \in \mathbb{V}, \forall r \in \mathbb{R}, \forall t \in \mathbb{T}, \forall u \in \mathbb{U}$

$\sum_{i \in \mathbb{V}} \sum_{j \in \mathbb{V}}\left(E_{i j} \cdot x_{i j}^{r t u}\right) \leq Q, \quad \forall r \in \mathbb{R}, \forall t \in \mathbb{T}, \forall u \in \mathbb{U}$

RemainEnergy $^{r t u}=Q-\sum_{i \in \mathbb{V}} \sum_{j \in \mathbb{V}}\left(E_{i j} \cdot x_{i j}^{r t u}\right), \quad$ where $t=1, \forall r \in \mathbb{R}, \forall u \in \mathbb{U}$

RemainEnergy $^{r t u}=$ RemainEnergy $^{r(t-1) u}+$ Charge $^{r(t-1) u}-\sum_{i \in \mathbb{V}} \sum_{j \in \mathbb{V}}\left(E_{i j} \cdot x_{i j}^{r t u}\right)$,

$t \in \mathbb{T} \backslash\{1\}, \forall r \in \mathbb{R}, \forall u \in \mathbb{U}$

$-\left(\right.$ Charge $^{r t u}+$ RemainEnergy $\left.^{r t u}-Q\right) \leq M \cdot\left(1-\right.$ Recharge $\left.^{r t u}\right)$,

$\forall r \in \mathbb{R}, \forall t \in \mathbb{T}, \forall u \in \mathbb{U}$

$(Q \cdot \theta)-$ RemainEnergy $^{r t u} \leq M \cdot$ Recharge $^{r t u}, \quad \forall r \in \mathbb{R}, \forall t \in \mathbb{T}, \forall u \in \mathbb{U}$

RemainEnergy $^{r t u}+$ Charge $^{r t u} \leq Q, \quad \forall r \in \mathbb{R}, \forall t \in \mathbb{T}, \forall u \in \mathbb{U}$

Charge $^{r t u} \leq M \cdot$ Recharge $^{r t u}, \quad \forall r \in \mathbb{R}, \forall t \in \mathbb{T}, \forall u \in \mathbb{U}$

RemainEnergy $^{r t u}-(Q \cdot \theta) \leq M \cdot\left(1-\right.$ Recharge $\left.^{r t u}\right), \quad \forall r \in \mathbb{R}, \forall t \in \mathbb{T}, \forall u \in \mathbb{U}$

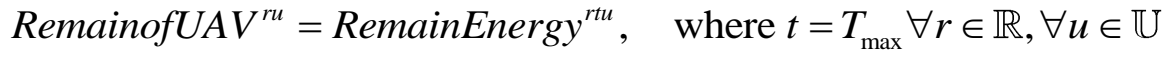

$\left(\sum_{i \in \mathbb{V}} \sum_{j \in \mathbb{V}}\left(d_{i j} / V\right) \cdot x_{i j}^{r t u}\right)+\left(\right.$ Charge $\left.^{r t u} \cdot R R\right)-$ TotalTime $^{r t u} \leq M \cdot\left(1-\right.$ Recharge $\left.^{r t u}\right)$,
$\forall r \in \mathbb{R}, \forall t \in \mathbb{T}, \forall u \in \mathbb{U}$

$\left(\sum_{i \in \mathbb{V}} \sum_{j \in \mathbb{V}}\left(d_{i j} / V\right) \cdot x_{i j}^{r t u}\right)-$ TotalTime $^{r t u} \leq M \cdot$ Recharge $^{r t u}$,
$\forall r \in \mathbb{R}, \forall t \in \mathbb{T} \backslash\left\{T_{\max }\right\}, \forall u \in \mathbb{U}$

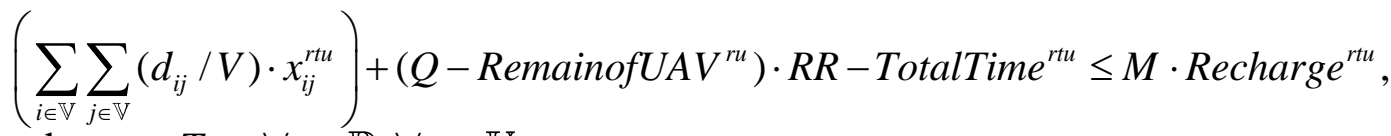

where $t=T_{\max }, \forall r \in \mathbb{R}, \forall u \in \mathbb{U}$

$\sum_{r \in \mathbb{R}} \sum_{t \in \mathbb{T}}$ TotalTime $^{r t u}-$ MaxTime $^{u} \leq T_{c}, \forall u \in \mathbb{U}$ 
MaxTime $^{u} \geq 0, \quad \forall u \in \mathbb{U}$

$\sum_{r \in \mathbb{R}} \sum_{t \in \mathbb{T}} \sum_{j \in \mathbb{V}} x_{0 j}^{r t u} \geq 1-M \cdot\left(1-\right.$ UAVUSed $\left.^{u}\right), \quad \forall u \in \mathbb{U}$

$\sum_{r \in \mathbb{R}} \sum_{t \in \mathbb{T}} \sum_{j \in \mathbb{V}} x_{0 j}^{r t u} \leq M \cdot$ UAVUsed $^{u}, \quad \forall u \in \mathbb{U}$

$E_{i j}=d_{i j} \cdot C R, \quad \forall(i, j) \in \mathbb{E}$

$1 \leq \mu_{i}^{r t u} \leq N, \quad \forall i \in \mathbb{V}, \forall r \in \mathbb{R}, \forall t \in \mathbb{T}, \forall u \in \mathbb{U}$

$\mu_{i}^{r t u}-\mu_{j}^{r t u}+N \cdot x_{i j}^{r t u} \leq N-1, \quad \forall(i, j) \in \mathbb{E}, \forall r \in \mathbb{R}, \forall t \in \mathbb{T}, \forall u \in \mathbb{U}$

Constraints (2) through (6) are basic constraints of the route planning problem:

- Constraint (2) prevents the UAVs from having self-loops in their routes.

- Constraints (3) and (4) ensure that every location is visited by any UAV exactly once.

- Constraint (5) forces the UAVs to fly at least one trip and ensure that all trips start and end at the base location.

- Constraint (6) ensures that each UAV arrives and departs location $i$ that it visits.

Constraint (7), adopted from the capacitated vehicle routing problem (CVRP), ensures that the energy consumed by each UAV in each trip cannot exceed the UAV's battery capacity.

Constraints (8) through (15) are specific to our problem to handle the energy consumption of UAVs:

- If the UAV is deployed in the first trip of each round, constraint (8) ensures that the current capacity is equal to the total capacity minus the amount of energy consumption to the next location.

- Constraint (9) calculates the remaining energy of each UAV for the remaining trips (except the first trip) of each round, whether the UAV has been recharged or not.

- Constraints (10) and (11) state that if the recharging occurs, the charging amount equals the battery capacity minus the remaining energy.

- Constraint (12) ensures that the sum of the remaining energy and the charging amount does not exceed the capacity.

- Constraints (13) and (14) decide that if the remaining energy is more than the limit capacity of each UAV, then the amount of charging equals zero.

- Constraint (15) tracks the remaining battery capacity of the last trip in each round of UAV $u$.

Constraints (16) through (20) are used to track the time spent by UAVs:

- Constraint (16) considers whether the UAV has been recharged or not. If the UAV has been recharged, the total time of the UAV equals the time consumed plus the recharging time, which is calculated from the amount of charging times the recharge rate of the UAV. 
- Constraint (17) considers the case that the UAV has not been recharged in each trip except for the last trip of each round, in which the total time of the UAV equals the time consumed by visiting locations.

- Constraint (18) considers the case that the UAV has not been recharged in the last trip of each round, in which the total time of the UAV equals the time consumed plus the charging time to fulfill the battery capacity.

- Constraint (19) calculates the penalty of excess mission time of each UAV.

- Constraint (20) forces the penalty of excess mission time to be non-negative.

Constraint (21) and (22) determine if UAV $u$ is used in the mission. Constraint (23) indicates the energy expense from location $i$ to $j$, which is calculated from distance from $i$ to $j$ and the energy consumption rate of the UAV. Constraints (24) and (25) are the subtour elimination constraints, which are used to give orders to all visited locations, excluding the base location (Miller et al., 1960).

\section{Numerical Experiments}

The optimization model formulated in the previous section is solved by using the branch-andbound algorithm in IBM ILOG CPLEX Optimization Studio Version 12.10.0.0 (International Business Machine, 2019), running on Ubuntu 18.04 LTS with Intel® Xeon® ${ }^{\circledR} .6 \mathrm{GHz} 8$-core E52640 V3 CPU and VMware 500 GB hard disk. The total cost, flying time of each UAV, and the model runtimes from the solutions are reported under grid-network test problems and a real terrain map. A grid network, or Manhattan network, used for the test problems has $N$ grid points, where each point is a two-dimensional coordinate $(x, y)$, and the distance between two adjacent grid points is $1,000 \mathrm{~m}$. The goals of the experiments are to study the effects of the model parameters and whether the proposed model can yield optimal solutions under various inputs. So, we varied the model parameters over a large number of combinations to explore the effects of model parameters and the model limitations. For the objective function to have a monetary cost unit, we set the energy unit cost $\gamma$ to the electricity billing rate (baht per mWh), the UAV unit $\operatorname{cost} \beta$ as the rental cost per UAV per day. For the computational resource constraints, we limit the CPU time and hard disk resources used for model solving to 48 hours and $500 \mathrm{~GB}$, respectively. The model will be prematurely terminated if either one of these two conditions is met, and the current best solution is taken as the final (non-optimal) solution.

\subsection{Significance of Model Parameters}

To determine which model parameters have significant effects on the costs, we design a singlereplicate two-level factorial experiment for five model parameters (factors A, B, C, D, E): the number of grid points, the number of available UAVs, the target mission time, the number of maximum allowed trips, and the number of required rounds. We fix the remaining model parameters as shown in Table 4. The responses of the experiments are the total cost and the individual cost components in the total cost.

Regarding the objective function coefficients, we set the energy unit cost $\gamma$ to the electricity billing rate of 0.0039 baht per mWh, based on Metropolitan Electricity Authority (MEA) (2020), and the UAV unit cost $\beta$ to the rental cost of 166.7 per UAV per day based on the market rates by to Drone Robotics Thailand (2020). We fixed the time penalty unit cost $\alpha$ to $0.3 \mathrm{baht} / \mathrm{second}$. The sensitivity of these coefficients on the optimal solution will be further investigated in Section 4.3. 
The number of UAVs and grid points used in the experiments are based on the last-mile parcel delivery application from the work of Aurambout et al. (2019). A set of 12 (4 by 3) and 20 (5 by 4) squared grid points with $1000 \mathrm{~m}$ between two adjacent grids were used as input maps in our experiments, with two and five UAVs available for deployment. We used one and three rounds to explore its effects and model limitations. The parameters related to the UAV are based on the specifications of the DJI Spark model (DJI, 2020), and the target mission time is chosen based on the size of the map, the importance of the mission time, and the UAV capabilities. For example, all the parcels are expected to be delivered within an hour for the coverage area between 12 and $20 \mathrm{~km}^{2}$.

Table 4. Model parameters for two-level factorial experiments.

\begin{tabular}{|l|l|}
\hline Varied Parameters & Values \\
\hline$N$ - Number of grid points & 12,20 \\
\hline$N_{v}$ - Number of available UAVs & 2,5 \\
\hline$T_{c}$ - Target mission time & None, 60 minutes \\
\hline$T_{\text {max }}$ - Number of maximum allowed trips for each UAV & 2,5 \\
\hline$N_{R}$ - Number of required rounds & 1,3 \\
\hline Fixed Parameters & Values \\
\hline$\alpha$ - Time penalty unit cost & 0.3 baht/second \\
\hline$\beta$ - UAV unit cost & 166.7 baht/UAV per day) \\
\hline$\gamma$ - Energy unit cost & 0.0039 baht $/ \mathrm{mWh}$ \\
\hline$Q$ - Battery capacity & 16872 watt-hours \\
\hline$V$ - UAV velocity & 13.86 meter/second \\
\hline$C R$ - Battery consumption rate & 1.26819 watt-hour/meter \\
\hline$R R$ - Battery recharging rate & 0.18492 seconds/watt-hour \\
\hline
\end{tabular}

Table 5 shows the experimental results from the single-replicate $2^{5}$ factorial experiment based on the model parameters in Table 4 . The results obtained for the number of grid points equal to 20 are sub-optimal due to the $500 \mathrm{~GB}$ hard disk limit constraint. The normal probability plot of the effect estimates based on the total cost in Figure 2(a) reveals that many effect estimates take the same value at zero and large negative effect estimates on the left. Due to the nature of the problem, the penalty cost behaves like a piecewise function that is only positive when the total mission time exceeds the target mission time, and the UAV cost behaves like a discrete step function of the number of UAVs used. So, the normality assumption of the total cost is not justified, and it is inappropriate to draw conclusions about the factor effects by using the normal probability plot. The normal probability plots of the effect estimates based on the penalty cost and the UAV cost (not shown) also look similar to the one in Figure 2(a).

On the other hand, the energy cost increases with the flying distance and takes on continuous values that are neither piecewise nor discrete. As such, we can draw conclusions regarding the effects of model parameters on the energy cost from our experiments. As shown in Figure 2(b), the normal probability plot of the effect estimates based on the energy cost reveals that the number of grid points (Factor A), the number of required rounds (Factor E), and their interaction are significant. In other words, the energy cost increases with both the number of grid points and the number of rounds. The positive interaction between the two factors means that the effect of the number of grid points on the energy cost is higher when the number of rounds increases. 
Table 5. Results from the single-replicate $2^{5}$ factorial experiment.

\begin{tabular}{|c|c|c|c|c|c|c|c|c|c|}
\hline $\begin{array}{l}\text { Ca } \\
\text { se }\end{array}$ & $\begin{array}{l}\text { Grid Points } \\
\text { (A) }\end{array}$ & $\begin{array}{c}\text { UAV } \\
\text { (B) }\end{array}$ & $\begin{array}{c}\text { Mission } \\
\text { Time (C) }\end{array}$ & $\begin{array}{l}\text { Max Allowed } \\
\text { Trips (D) }\end{array}$ & $\begin{array}{c}\text { Round } \\
\text { (E) }\end{array}$ & $\begin{array}{l}\text { Energy } \\
\text { Cost }\end{array}$ & $\begin{array}{l}\text { Penalty } \\
\text { Cost }\end{array}$ & $\begin{array}{l}\text { UAV } \\
\text { Cost }\end{array}$ & $\begin{array}{l}\text { Total } \\
\text { Cost }\end{array}$ \\
\hline 1 & 12 & 2 & 0 & 2 & 1 & 59.35 & 0 & 166.7 & 226.05 \\
\hline 2 & 20 & 2 & 0 & 2 & 1 & 105.91 & 0 & 166.7 & 272.61 \\
\hline 3 & 12 & 5 & 0 & 2 & 1 & 59.35 & 0 & 166.7 & 226.05 \\
\hline 4 & 20 & 5 & 0 & 2 & 1 & 105.91 & 0 & 166.7 & 272.61 \\
\hline 5 & 12 & 2 & 3600 & 2 & 1 & 59.35 & 23.998 & 166.7 & 250.048 \\
\hline 6 & 20 & 2 & 3600 & 2 & 1 & 105.91 & 0 & 333.4 & 439.31 \\
\hline 7 & 12 & 5 & 3600 & 2 & 1 & 59.35 & 23.998 & 166.7 & 250.048 \\
\hline 8 & 20 & 5 & 3600 & 2 & 1 & 105.91 & 0 & 333.4 & 439.31 \\
\hline 9 & 12 & 2 & 0 & 5 & 1 & 59.35 & 0 & 166.7 & 226.05 \\
\hline 10 & 20 & 2 & 0 & 5 & 1 & 105.91 & 0 & 166.7 & 272.61 \\
\hline 11 & 12 & 5 & 0 & 5 & 1 & 59.35 & 0 & 166.7 & 226.05 \\
\hline 12 & 20 & 5 & 0 & 5 & 1 & 105.91 & 0 & 166.7 & 272.61 \\
\hline 13 & 12 & 2 & 3600 & 5 & 1 & 59.35 & 23.998 & 166.7 & 250.048 \\
\hline 14 & 20 & 2 & 3600 & 5 & 1 & 105.91 & 0 & 333.4 & 439.31 \\
\hline 15 & 12 & 5 & 3600 & 5 & 1 & 59.35 & 23.998 & 166.7 & 250.048 \\
\hline 16 & 20 & 5 & 3600 & 5 & 1 & 105.91 & 0 & 333.4 & 439.31 \\
\hline 17 & 12 & 2 & 0 & 2 & 3 & 178.05 & 0 & 166.7 & 344.75 \\
\hline 18 & 20 & 2 & 0 & 2 & 3 & 317.74 & 0 & 333.4 & 651.14 \\
\hline 19 & 12 & 5 & 0 & 2 & 3 & 178.05 & 0 & 166.7 & 344.75 \\
\hline 20 & 20 & 5 & 0 & 2 & 3 & 317.74 & 0 & 333.4 & 651.14 \\
\hline 21 & 12 & 2 & 3600 & 2 & 3 & 178.05 & 1152 & 333.4 & 1663.45 \\
\hline 22 & 20 & 2 & 3600 & 2 & 3 & 317.74 & 3750.3 & 333.4 & 4401.44 \\
\hline 23 & 12 & 5 & 3600 & 2 & 3 & 182.15 & 148.21 & 500.1 & 830.46 \\
\hline 24 & 20 & 5 & 3600 & 2 & 3 & 324.73 & 700.21 & 833.5 & 1858.44 \\
\hline 25 & 12 & 2 & 0 & 5 & 3 & 178.05 & 0 & 166.7 & 344.75 \\
\hline 26 & 20 & 2 & 0 & 5 & 3 & 317.74 & 0 & 333.4 & 651.14 \\
\hline 27 & 12 & 5 & 0 & 5 & 3 & 178.05 & 0 & 166.7 & 344.75 \\
\hline 28 & 20 & 5 & 0 & 5 & 3 & 317.74 & 0 & 333.4 & 651.14 \\
\hline 29 & 12 & 2 & 3600 & 5 & 3 & 178.05 & 1152 & 333.4 & 1663.45 \\
\hline 30 & 20 & 2 & 3600 & 5 & 3 & 317.74 & 3750.3 & 333.4 & 4401.44 \\
\hline 31 & 12 & 5 & 3600 & 5 & 3 & 182.15 & 148.21 & 500.1 & 830.46 \\
\hline 32 & 20 & 5 & 3600 & 5 & 3 & 324.73 & 700.21 & 833.5 & 1858.44 \\
\hline
\end{tabular}

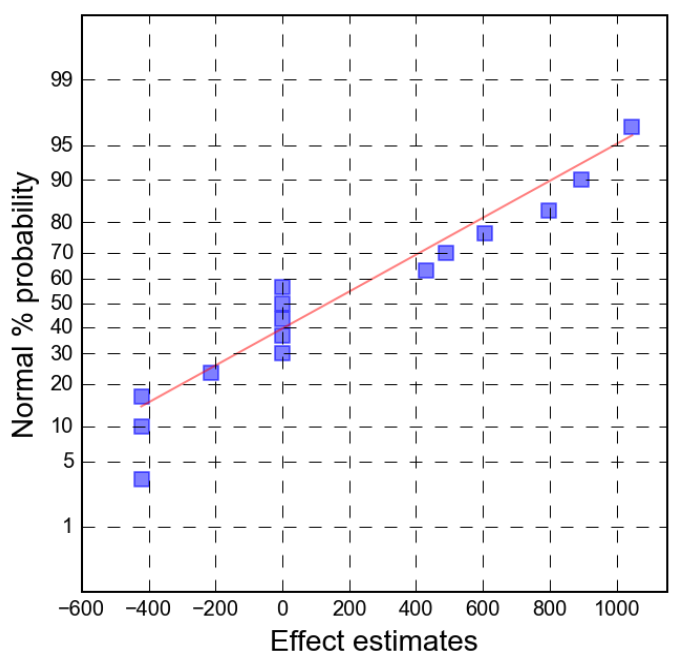

(a) Total cost as the response.

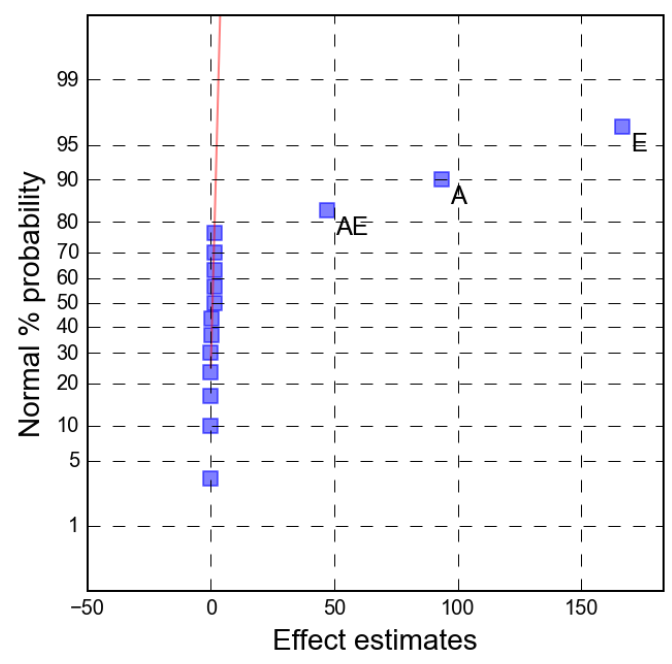

(b) Energy cost as the response.

Figure 2. Normal probability plots of effect estimates based on the total cost and the energy cost. 


\subsection{Exploring Effects of Model Parameters}

To better understand the effects of other model parameters such as the number of points to visit and the time penalty unit cost, the following test problems shown in Table 6 are generated:

- Cases 1 to 5 represent a small deployment area with two UAVs available, where 12 grid points are used. The target mission time is fixed to 1,800 seconds, and the effect of time penalty unit cost $\alpha$ is studied by varying it as $0,0.01,0.1,0.2$, and 0.3 baht per second.

- Case 6 is the same as case 5 except that the number of available UAVs is increased from 2 to 5 .

- Cases 7 to 11 represent a larger deployment area with two UAVs available, where 20 grid points are used. The target mission time is changed to 3,600 seconds, and the effect of time penalty unit cost $\alpha$ is studied by varying it as $0,0.01,0.1,0.2$, and 0.3 baht per second.

- Case 12 is the same as case number 11 except that the number of available UAVs is increased from 2 to 5 .

The maximum allowed trips per UAV and the number of required rounds are two trips and one round, respectively. The energy unit cost $\gamma$ is kept to 0.0039 baht per mWh based on the actual electricity billing rate from Metropolitan Electricity Authority of Thailand, and the UAV unit cost $\beta$ is fixed to 166.7 baht per UAV (per day) based on the market rates by Drone Robotics Thailand (2020).

Table 6 shows the results obtained on the above test problems. The row Optimality status indicates whether the solution is optimal or sub-optimal due to CPU time limit or disk space limit. We observe that the optimal solutions are found under less than two hours only in the case of 12 grid points. When the number of available UAVs increases to five or the number of grid points is increased to 20, the model cannot be solved within the 48-hour CPU time limit or 500 GB disk space limit. Another observation is the relationship between the time penalty unit cost and the number of UAVs involved. For example, if the time penalty unit cost gets higher, as in cases 3 to 5 , the model will add another UAV to decrease the time penalty cost.

From Table 7, cases 13-16 and 17-18, respectively, aim to explore the effects of the number of rounds and the UAV unit cost. For cases 13-16, the number of maximum allowed trips is set to five. Allowing more trips per UAV leads to a longer time to achieve an optimal solution, even though it yields the same total cost compared to case number 5 in Table 6 . The reason is that allowing more trips adds more options and hence increases the search space for the model. The results in cases 13-16 show that the number of required rounds directly affects the total cost and the disk space usage. The available computing resources could not solve the model if the number of rounds is set to three. When the UAV unit cost increased from 300 to $500 \mathrm{baht} / \mathrm{second}$ (cases 17 and 18), the model will reduce the number of UAVs used in the mission to minimize the total cost. 
Phalapanyakoon \& Siripongwutikorn: Route Planning of Unmanned Aerial Vehicles under Recharging...

Table 6. Results on the grid problems with two maximum allowed trips and one round.

\begin{tabular}{|c|c|c|c|c|c|c|c|c|c|c|c|c|c|}
\hline & & \multicolumn{12}{|c|}{ Case No. } \\
\hline & & 1 & 2 & 3 & 4 & 5 & 6 & 7 & 8 & 9 & 10 & 11 & 12 \\
\hline \multirow{7}{*}{$\begin{array}{c}\text { Input } \\
\text { Parameters }\end{array}$} & \# UAVs & 2 & 2 & 2 & 2 & 2 & 5 & 2 & 2 & 2 & 2 & 2 & 5 \\
\hline & \# Grids & 12 & 12 & 12 & 12 & 12 & 12 & 20 & 20 & 20 & 20 & 20 & 20 \\
\hline & Target mission time (s) & 1800 & 1800 & 1800 & 1800 & 1800 & 1800 & 3600 & 3600 & 3600 & 3600 & 3600 & 3600 \\
\hline & Energy unit cost & 0.0039 & 0.0039 & 0.0039 & 0.0039 & 0.0039 & 0.0039 & 0.0039 & 0.0039 & 0.0039 & 0.0039 & 0.0039 & 0.0039 \\
\hline & Time penalty unit cost & 0 & 0.01 & 0.1 & 0.2 & 0.3 & 0.3 & 0 & 0.01 & 0.1 & 0.2 & 0.3 & 0.3 \\
\hline & UAV unit cost & 166.7 & 166.7 & 166.7 & 166.7 & 166.7 & 166.7 & 166.7 & 166.7 & 166.7 & 166.7 & 166.7 & 166.7 \\
\hline & Optimality status & optimal & optimal & optimal & optimal & optimal & $\begin{array}{l}\text { time } \\
\text { limit }\end{array}$ & $\begin{array}{l}\text { HDD } \\
\text { limit }\end{array}$ & $\begin{array}{l}\text { HDD } \\
\text { limit }\end{array}$ & $\begin{array}{l}\text { HDD } \\
\text { limit }\end{array}$ & $\begin{array}{l}\text { HDD } \\
\text { limit }\end{array}$ & $\begin{array}{l}\text { HDD } \\
\text { limit }\end{array}$ & $\begin{array}{l}\text { HDD } \\
\text { limit }\end{array}$ \\
\hline \multirow{4}{*}{ Costs } & Energy cost & 59.35 & 59.35 & 59.35 & 66.35 & 66.35 & 66.35 & 105.91 & 105.91 & 105.91 & 105.91 & 105.91 & 105.91 \\
\hline & Time penalty cost & - & 18.8 & 188 & 102.74 & 154.1 & 154.1 & - & 29.67 & 0 & 0 & 0 & 0 \\
\hline & UAV cost & 166.7 & 166.7 & 166.7 & 333.4 & 333.4 & 333.4 & 166.7 & 166.7 & 333.4 & 333.4 & 333.4 & 333.4 \\
\hline & Total Cost & 226.05 & 244.85 & 414.05 & 502.49 & 553.85 & 553.85 & 272.61 & 302.28 & 439.31 & 439.31 & 439.31 & 439.31 \\
\hline \multirow{6}{*}{$\begin{array}{l}\text { Total Time } \\
\text { Consumed } \\
\text { Per UAV }\end{array}$} & UAV1 & 0 & 3679.99 & 0 & 1840 & 2273.69 & 0 & 0 & 0 & 3066.66 & 3500.35 & 3500.35 & 0 \\
\hline & UAV2 & 3679.99 & 0 & 3679.99 & 2273.69 & 1840 & 0 & 6567.01 & 6567.01 & 3500.35 & 3066.66 & 3066.66 & 0 \\
\hline & UAV3 & - & - & - & - & - & 2273.69 & - & - & - & - & - & 3500.35 \\
\hline & UAV4 & - & - & - & - & - & 0 & - & - & - & - & - & 3066.66 \\
\hline & UAV5 & - & - & - & - & - & 1840 & - & - & - & - & - & 0 \\
\hline & CPU Runtime (h) & 0.75 & 1.72 & 0.81 & 0.05 & 0.01 & 48 & 10.04 & 21.49 & 16.85 & 19.59 & 24.11 & 6.89 \\
\hline
\end{tabular}

Table 7. Results on the grid problems with different maximum allowed trips and rounds.

\begin{tabular}{|c|c|c|c|c|c|c|c|}
\hline & & \multicolumn{6}{|c|}{ Case No. } \\
\hline & & 13 & 14 & 15 & 16 & 17 & 18 \\
\hline \multirow{7}{*}{ Input Parameters } & \# UAVs & 2 & 2 & 2 & 5 & 2 & 2 \\
\hline & \# Grids & 12 & 12 & 12 & 12 & 12 & 12 \\
\hline & \# Maximum allowed trips & 5 & 5 & 5 & 5 & 2 & 2 \\
\hline & Target mission time $(\mathrm{s})$ & 1800 & 1800 & 1800 & 1800 & 1800 & 1800 \\
\hline & \# Rounds & 1 & 2 & 3 & 3 & 1 & 1 \\
\hline & Time penalty unit cost & 0.3 & 0.3 & 0.3 & 0.3 & 0.3 & 0.3 \\
\hline & UAV unit cost & 166.7 & 166.7 & 166.7 & 166.7 & 300 & 500 \\
\hline & Optimality Status & optimal & optimal & HDD limit & HDD limit & optimal & optimal \\
\hline \multirow{4}{*}{ Costs } & Energy cost & 66.35 & 118.7 & 178.05 & 192.04 & 66.35 & 59.35 \\
\hline & Time penalty cost & 154.1 & 1128 & 2232 & 872.21 & 154.1 & 564 \\
\hline & UAV cost & 333.4 & 333.4 & 333.4 & 833.5 & 600 & 500 \\
\hline & Total Cost & 553.85 & 1580.1 & 2743.45 & 1897.75 & 820.45 & 1123.35 \\
\hline \multirow{6}{*}{$\begin{array}{c}\text { Total Time Consumed } \\
\text { Per UAV }\end{array}$} & UAV1 & 2273.69 & 3679.99 & 7359.98 & 3679.99 & 2273.69 & 3679.99 \\
\hline & UAV2 & 1840 & 3679.99 & 3679.99 & 1840 & 1840 & 0 \\
\hline & UAV3 & - & - & - & 1840 & - & - \\
\hline & UAV4 & - & - & - & 2273.69 & - & - \\
\hline & UAV5 & - & - & - & 2273.69 & - & - \\
\hline & CPU Runtime (h) & 0.03 & 8.01 & 18.82 & 11.25 & 0.16 & 1.69 \\
\hline
\end{tabular}


From the experimental results on the grid networks in this section, we can conclude the following:

- Due to computational limitations, the model cannot handle problem instances with a large number of points to visit or a large number of rounds.

- The time penalty unit cost and the UAV unit cost have an opposite effect on the number of UAVs to be deployed. The increase of the time penalty unit cost will lead to more UAVs being used to reduce the total mission time, while the increase of the UAV unit cost may lead to fewer UAVs being used, at the expense of a higher mission time. For a time-critical mission, using a high time penalty unit cost is preferable to get a low total mission time.

\subsection{Sensitivity Analysis}

Since our model is a mixed-integer one, we use a graphical method for sensitivity analysis to study how the optimal solution is affected by changes in the model parameters, including the problem's objective function coefficients and the right-hand sides of the constraints. Twelve grid points with 2 UAVs and a maximum allowed trips per UAV of 2 were implemented in both cases. The number of required rounds was set at 1 with a target mission time of 1,800 seconds. From (1), the problem's objective function coefficients are the energy unit cost $(\gamma)$, the time penalty unit cost $(\alpha)$, and the UAV unit cost $(\beta)$. We fixed one of the coefficients at the time and varied the other two coefficients around their default value so that the optimal solutions or total costs as a function of the two varied coefficients can be visualized. The following three cases are used:

- Case 1: Fixed: Energy unit cost of $0.0039 \mathrm{baht} / \mathrm{mWh}$

Varied: (1) Time penalty unit cost $[0,0.05,0.1,0.3,0.5]$

(2) UAV unit cost $[166.7,200,250,300,350]$

- Case 2: Fixed: Time penalty unit cost of $0.3 \mathrm{baht} / \mathrm{second}$

Varied: (1) Energy unit cost [0.003, 0.004, 0.005, 0.006, 0.007]

(2) UAV unit cost [166.7, 200, 250, 300, 350]

- Case 3: Fixed: UAV unit cost of 166.7 baht/UAV

Varied: (1) Time penalty unit cost $[0,0.05,0.1,0.3,0.5]$

(2) Energy unit cost $[0.003,0.004,0.005,0.006,0.007]$

In each case, the optimal solutions are interpolated to obtain a smooth surface, as shown in Figure 3. From Figure 3(a), it can be observed that the UAV unit cost and the time penalty unit cost appears to have a synergetic effect on the total cost. Namely, the total cost increases faster with the time penalty unit cost at the higher UAV unit cost and vice versa. When the UAV unit cost is high, the model may decide to use only a few UAVs, which prolongs the mission time and amplifies the effect of the time penalty cost.

The UAV unit cost and the energy unit cost appear to act relatively independently on the total cost, as shown in Figure 3(b). Changing the UAV unit cost does not affect how the total cost increases with the energy unit cost, as the total flying distance remains the same. Also, the total cost is marginally affected by the increase of the energy unit cost, as the energy cost contributes only a small portion of the total cost. Finally, the time penalty unit cost significantly affects the 
total cost for a given UAV unit cost, as shown in Figure 3(b), independent of the energy unit cost. In summary, among the three objective function coefficients, the total cost is most sensitive to the time penalty unit cost but least sensitive to the energy unit cost, and there exists the synergetic effect between the time penalty unit cost and the UAV unit cost.

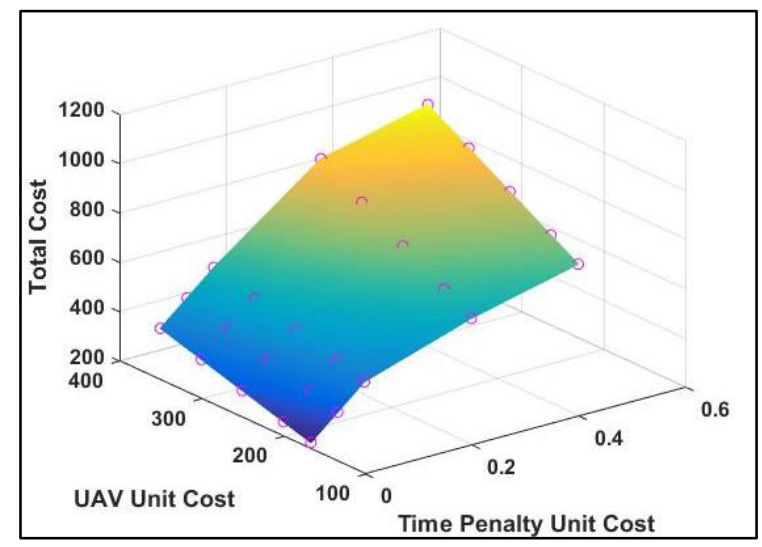

(a) Case 1: Effect of time penalty unit cost and UAV unit cost (Energy unit cost $0.0039 \mathrm{baht} / \mathrm{mWh}$ ).

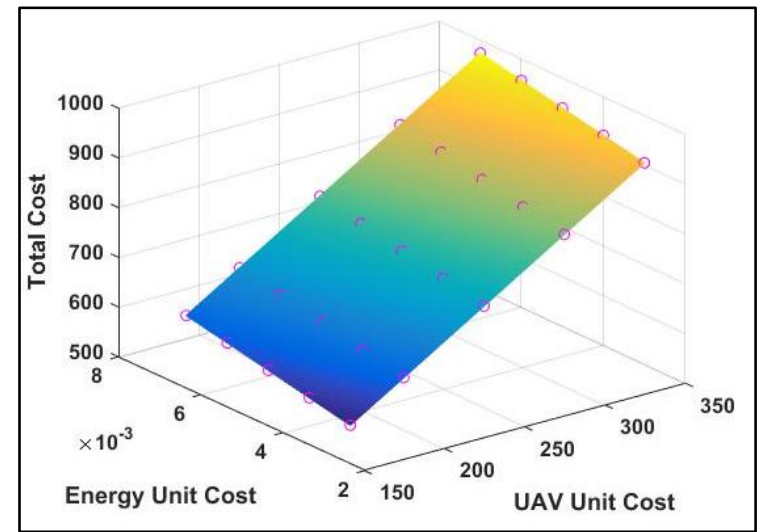

(b) Case 2: Effect of energy unit cost and UAV unit cost (Time penalty unit cost 0.3 baht/second).

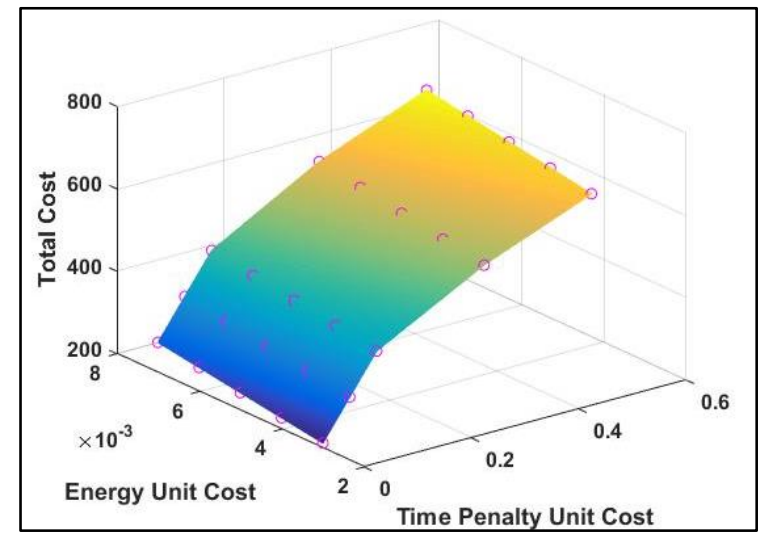

(c) Case 3: Effect of energy unit cost and Time penalty unit cost (UAV unit cost 166.7 baht/UAV).

Figure 3. Sensitivity analysis on the coefficients of the objective function. 
The parameters for the right-hand sides of the constraints are the Battery Capacity $(Q)$ from constraint (7), Target Mission Time $\left(T_{c}\right)$ from constraint (19), and Energy Consumption Rate $(C R)$ from constraint (23). The effects of these three parameters on the total cost are shown in Figure 4. From Figure 4(a), too low battery capacities result in an infeasible solution because UAVs cannot their trip. When the battery capacity is sufficiently high, the UAVs will be able to finish their trips. However, they need many returns to the base for recharging, resulting in a high total cost. As the battery capacity increases, the UAVs will need to return less and less to the base for recharging, hence a smaller total cost. If the battery capacity increases beyond a certain threshold, it will no longer reduce the total cost because the UAVs can complete the mission without any recharging, minimizing the energy cost component in the total cost to zero. The effect of the target mission time on the total cost is straightforward, as shown in Figure 4(b). The total cost decreases with the target mission time until the time penalty cost component in the total cost reaches zero, and the total cost is no longer affected by the target mission time. The effect of the energy consumption rate on the total cost is opposite of that of the target mission time. However, too high an energy consumption rate leads to an infeasible solution because the energy consumption of each UAV exceeds its battery capacity to finish the mission.

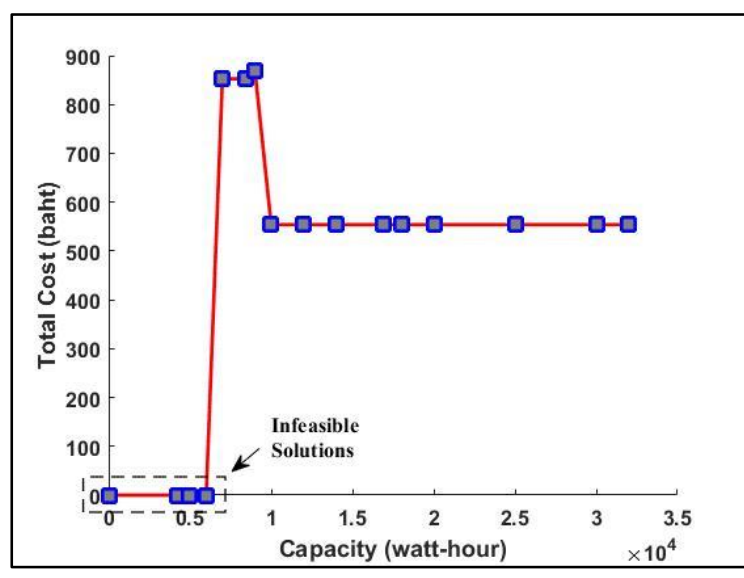

(a) Battery Capacity $(Q)$

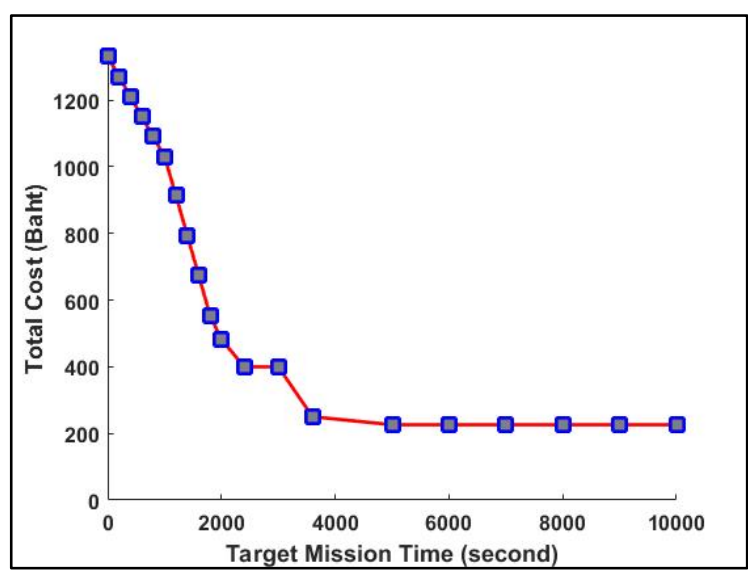

(b) Target Mission Time $\left(T_{c}\right)$

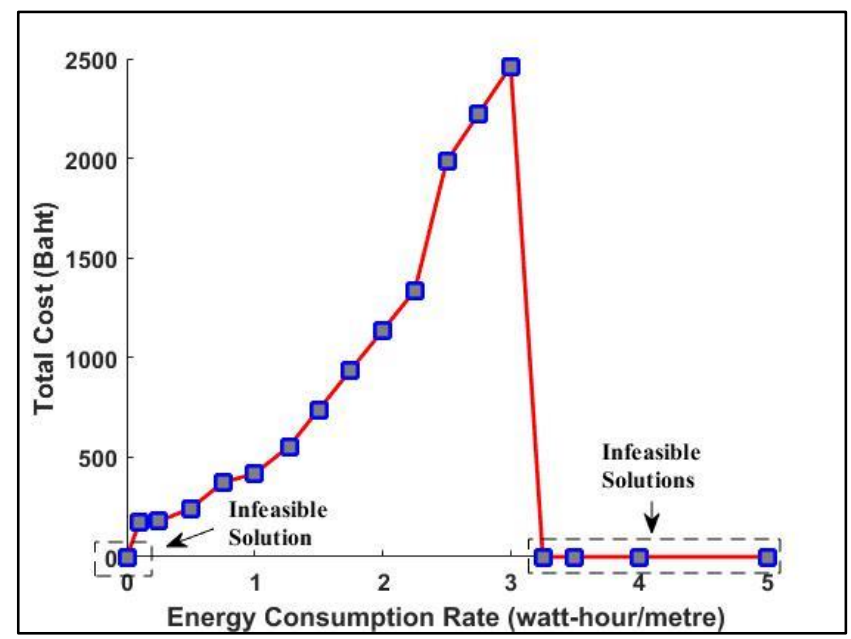

(c) Energy Consumption Rate $(C R)$

Figure 4. Sensitivity analysis on the right-hand sides of the constraints. 


\subsection{Experimental Results on Real Terrain}

We also experimented on a real terrain, which is a part of the downtown area of Nong Khai province, Thailand, to examine the feasibility and computational performance of our proposed model and see whether it shares the same behavior as in the grid network studied in the previous section. First, the raw map is obtained from Google Maps, and the map is manually discretized into 25-by-15 grid points, where the distance of two adjacent grid points is 130 meters. Then, 15 and 30 grid points are manually specified as the locations to visit to explore the model limitations on the number of grid points of a real terrain problem. The time penalty unit cost is varied as 0.3 and $0.6 \mathrm{baht} / \mathrm{second}$ to emphasize the mission that needs to be finished quickly. The target mission time is set to 720 and 1440 seconds based on the number of grid points and the actual size of the area. Two of UAVs with the number of maximum allowed trip per UAV of 2 is considered. Only one round is considered in this mission for all cases. The energy unit cost and UAV unit cost are 0.0039 baht per $\mathrm{mWh}$ and 166.7 baht per UAV, respectively.

The computational results from four cases are shown in Table 8, with flying paths and timing diagrams shown in Figure 5. The time penalty unit cost affects the number of UAVs used. For the case of 15 grid points (cases 1 and 2), the lower time penalty unit cost ( 0.3 baht/second) leads to only one UAV being used at the expense of longer mission time, while the higher time penalty unit cost ( 0.6 baht/second) results in two UAVs being used. In either case, the completion times exceed the target mission times, but the latter case completes in less time as more UAVs are deployed. The results of case 1 and case 2 in Table 8 suggest that, for a time-sensitive mission, the solution with a higher total cost may be preferred if it gives a lower time penalty cost. However, when we implement 30 grid points in the experiments (cases 3 and 4), the model cannot be solved within the 48-hour CPU time limit.

Table 8. Costs and runtimes on real terrain area.

\begin{tabular}{|c|c|c|c|c|c|}
\hline & & \multicolumn{4}{|c|}{ Case No. } \\
\hline & & 1 & 2 & 3 & 4 \\
\hline \multirow{2}{*}{ Input Parameters } & \# grids & 15 & 15 & 30 & 30 \\
\hline & Time penalty unit cost & 0.3 & 0.6 & 0.3 & 0.6 \\
\hline & Optimality Status & Optimal & optimal & time limit & time limit \\
\hline \multirow{4}{*}{ Cost } & Energy cost & 29.32 & 32 & 59.44 & 59.44 \\
\hline & Time penalty cost & 315.81 & 296.53 & 214.06 & 428.12 \\
\hline & UAV cost & 166.7 & 333.4 & 333.4 & 333.4 \\
\hline & Total Cost & 511.83 & 661.93 & 606.90 & 820.96 \\
\hline \multirow{2}{*}{ Total Time Consumed Per UAV } & UAV1 & 1772.70 & 924.83 & 2055.90 & 1537.70 \\
\hline & UAV2 & 0.00 & 1009.38 & 1537.70 & 2055.90 \\
\hline \multicolumn{2}{|c|}{ CPU Runtime (h) } & 4.83 & 0.05 & 48 & 48 \\
\hline
\end{tabular}



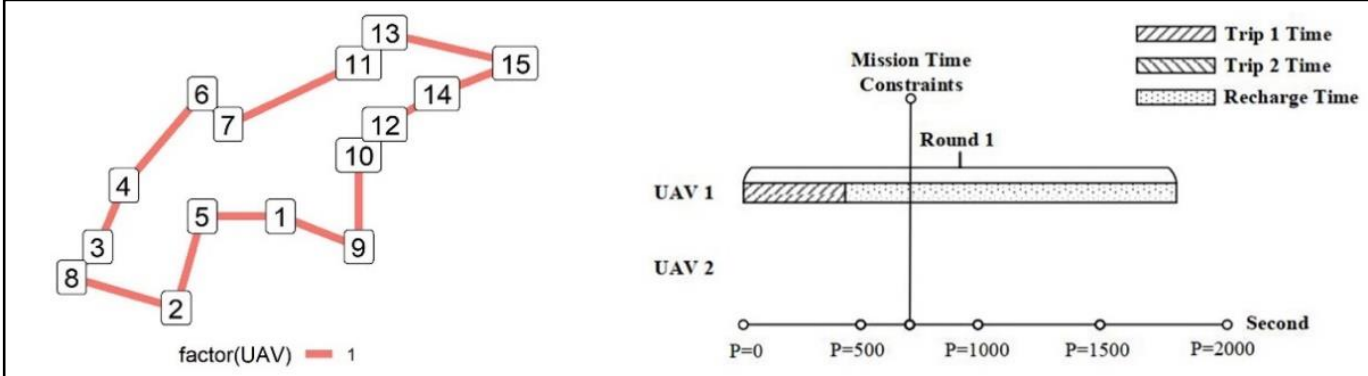

(a) Time penalty unit cost $0.3 \mathrm{baht} / \mathrm{second}$. One UAV is used with a total time of 1772.7 seconds for 15 grid points.

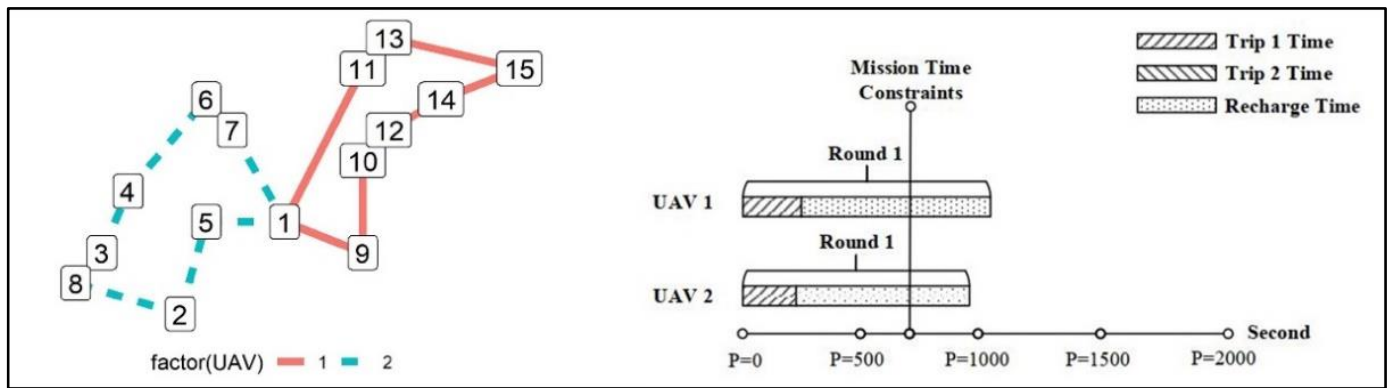

(b) Time penalty unit cost 0.6 baht/second. Two UAVs are used with total times of 1009.38 and 924.83 seconds for 15 grid points.

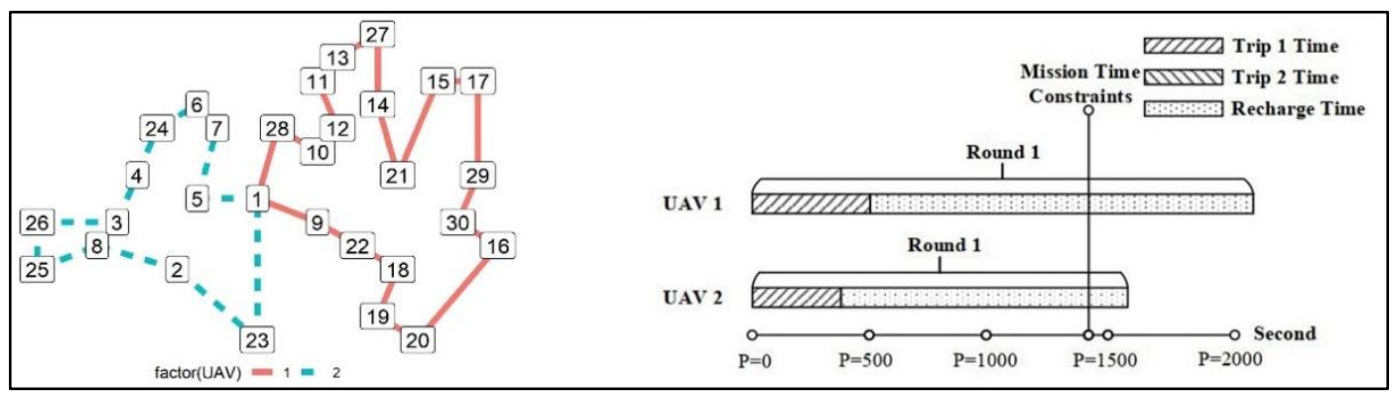

(c) Time penalty unit cost 0.3 baht/second. Two UAVs are used with total times of 2055.90 and 1537.70 seconds for 30 grid points.

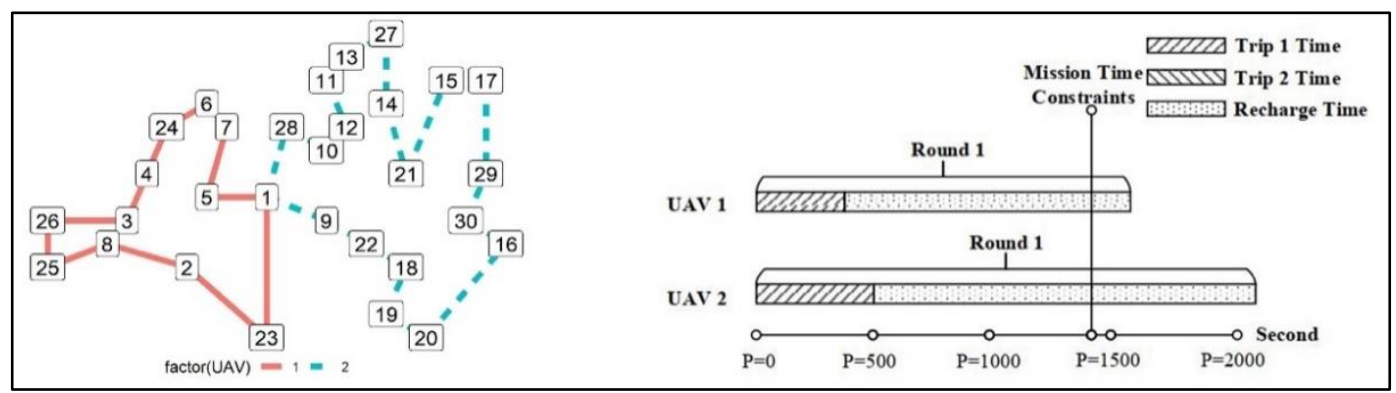

(d) Time penalty unit cost 0.6 baht/second. Two UAVs are used with total times of 1537.70 and 2055.90 seconds for 30 grid points.

Figure 5. Flying paths and timing diagrams for the real terrain scenario. 


\section{Conclusions}

In this research, the problem of route planning of multiple rechargeable UAVs flying multiple trips and rounds to cover the set of predefined locations under mission time constraints is introduced and studied as a novel approach for route planning of a rechargeable electric fleet of UAVs. Compared to existing works, the proposed model incorporates the UAV recharging period that depends on the UAV remaining energy when returning to the base, and the total cost is computed as the sum of the excess mission time, the electric cost contributed by the recharging, and the usage cost of deploying UAVs, which more accurately characterizes realistic scenarios. The problem is formulated as a mixed-integer model and solved under various settings of the model parameters with test problems and a real terrain area. The experimental results show that our mathematical model can obtain the optimal solutions for small problem sizes where only a moderate number of grid points and UAVs are considered. The model's ability to obtain optimal solutions for larger problem sizes is only limited by allocated CPU time and disk space. The number of available UAVs and the number of maximum allowed trips per UAV increase the search space, and there is a trade-off between the UAV unit cost and the time penalty unit cost. The model will decide whether it will deploy additional UAVs to reduce the maximum time of each UAV. Assigning more weight to the time penalty in the objective function may reduce the time penalty cost at the expense of using more UAVs, and hence a higher UAV usage cost. To demonstrate the model's practicality, the model was also studied in real terrain cases where the optimal solutions can be obtained under a network of 15 grid points. The sensitivity analysis of the objective function coefficients reveals that the total cost is most sensitive to the time penalty unit cost but least sensitive to the energy unit cost, and there exists the synergetic effect between the time penalty unit cost and the UAV unit cost.

For the research limitations, the proposed model only considers homogeneous UAVs. The UAV load-carrying capacity constraint that limits the number of visiting locations of each UAV is not considered. Also, the model is unable to yield optimal solutions if the network size or the number UAVs becomes large. The straightforward extension of this work is to integrate the fleet of heterogeneous UAVs and load-carrying capacity constraints to enhance the model's applicability in real-world situations. More importantly, heuristic methods must be explored to handle a large problem size.

Conflict of Interest

The authors confirm that there is no conflict of interest to declare for this publication.

Acknowledgments

This research is supported by the Petchra Pra Jom scholarship from King Mongkut's University of Technology Thonburi.

\section{References}

Alonso, F., Alvarez, M.J., \& Beasley, J.E. (2008). A tabu search algorithm for the periodic vehicle routing problem with multiple vehicle trips and accessibility restrictions. Journal of the Operational Research Society, 59, 963-976. https://doi.org/10.1057/palgrave.jors.2602405.

Aurambout, J.P., Gkoumas, K., \& Ciuffo, B. (2019). Last mile delivery by drones: an estimation of viable market potential and access to citizens across European cities. European Transport Research Review, 11(30), 1-21. https://doi.org/10.1186/s12544-019-0368-2. 
Authority, M.E. (2020). Energy charge of residential. URL: https://www.mea.or.th/en/profile/109/111.

Ayadi, R., \& Benadada, Y. (2013). Memetic algorithm for a multi-objective vehicle routing problem with multiple trips. International Journal of Computer Science and Applications, 10(2), 72-91.

Choi, Y., Robertson, B., Choi, Y., \& Mavris, D. (2019). A multi-trip vehicle routing problem for small unmanned aircraft systems-based urban delivery. Journal of Aircraft, 56(6), 2309-2323. https://doi.org/10.2514/1.C035473

Coelho, B.N., Coelho, V.N., Coelho, I.M., Ochi, L.S., Haghnazar, K.R., Zuidema, D., Lima, M.S.F., \& Da Costa, A.R. (2017). A multi-objective green UAV routing problem. Computers \& Operations Research, 88(1), 306-315. https://doi.org/10.1016/j.cor.2017.04.011.

Conrad, R.G., \& Figliozzi, M.A. (2011, May). The recharging vehicle routing problem. In Proceedings of the 2011 Industrial Engineering Research Conference, (pp. 8). IISE Norcross, GA.

Dantzig, G.B., \& Ramser, J.H. (1959). The truck dispatching problem. Management Science, 6(1), 80-91. https://doi.org/10.1287/mnsc.6.1.80.

DJI. (2020). Spark specs. URL: https://www.dji.com/spark/info\#specs.

Dorling, K., Heinrichs, J., Messier, G.G., \& Magierowski, S. (2017). Vehicle routing problems for drone delivery. In IEEE Transactions on Systems, Man, and Cybernetics: Systems, 47(1), 70-85. https://doi.org/10.1109/TSMC.2016.2582745.

Han, Y.Q., Li, J.Q., Liu, Z., Liu, C., \& Tian, J. (2020). Metaheuristic algorithm for solving the multiobjective vehicle routing problem with time window and drones. International Journal of Advanced Robotic Systems, 17(2). https://doi.org/10.1177/1729881420920031.

Hiermann, G., Puchinger, J., Ropke, S., \& Hartl, R.F. (2016). The electric fleet size and mix vehicle routing problem with time windows and recharging stations. European Journal of Operational Research, 252(3), 995-1018. https://doi.org/10.1016/j.ejor.2016.01.038.

International Business Machines Corporation. (2021, December). IBM ilog cplex optimization studio (Version 12.10.0.0). URL: https://www.ibm.com/products/ ilog-cplex-optimization-studio.

Joshi, D. (2019). Drone technology uses and applications for commercial, industrial and military drones in 2020 and the future. URL: https://www.businessinsider.com/drone-technology-uses-applications.

Kabcome, P., \& Mouktonglang, T. (2015). Vehicle routing problem for multiple product types, compartments, and trips with soft time windows. International Journal of Mathematics and Mathematical Sciences, 2015, 1-9. https://doi.org/10.1155/2015/126754.

Kitjacharoenchai, P., \& Lee, S. (2019). Vehicle routing problem with drones for last mile delivery. Procedia Manufacturing, 39, 314-324. https://doi.org/10.1016/j.promfg.2020.01.338.

Miller, C.E., Tucker, A.W., \& Zemlin, R.A. (1960). Integer programming formulation of traveling salesman problems. Journal of the ACM, 7(4), 326-329. https://doi.org/10.1145/321043.321046.

Penna, P.H.V., Afsar, H.M., Prins, C., \& Prodhon, C. (2016). A hybrid iterative local search algorithm for the electric fleet size and mix vehicle routing problem with time windows and recharging stations. IFAC-PapersOnLine, 49(12), 955-960. https: //doi.org/10.1016/j.ifacol.2016.07.899.

Thailand, D.R. (2020). Spark drone for rent. URL: https://www.facebook.com/dronerobotics.in.th/.

Troudi, A., Addouche, S.A., Dellagi, S., \& Mhamedi, A.E. (2018). Sizing of the drone delivery fleet considering energy autonomy. Sustainability, 10(9), 1-17. https://doi.org/10.3390/su10093344.

Yu, K., Budhiraja, A.K., Buebel, S., \& Tokekar, P. (2019). Algorithms and experiments on routing of unmanned aerial vehicles with mobile recharging stations. Journal of Field Robotics, 36(3), 602-616. https://doi.org/10.1002/rob.21856. 
Zhen, L., Ma, C., Wang, K., Xiao, L., \& Zhang, W. (2020). Multi-depot multi-trip vehicle routing problem with time windows and release dates. Transportation Research Part E: Logistics and Transportation Review, 135(1), 1-21. https://doi.org/10.1016/j.tre.2020.101866.

Zmazek, B., Taranenko, A., \& Smid, M. (2005). Capacitated VRP with time windows and multiple trips within working day. In 27th International Conference on Information Technology Interfaces, (pp. 104109). IEEE. Cavtat, Croatia. https://doi.org/10.1109/ITI.2005.1491105. 